\title{
Divisors of the Euler and Carmichael functions
}

\author{
by
}

KEvin Ford and Yong Hu (Urbana, IL)

Dedicated to Wolfgang Schmidt on the occasion of his 75th birthday

1. Introduction. Two of the most studied functions in the theory of numbers are Euler's totient function $\phi(n)$ and Carmichael's function $\lambda(n)$, the first giving the order of the group $(\mathbb{Z} / n \mathbb{Z})^{*}$ of reduced residues $\bmod$ ulo $n$, and the latter giving the maximum order of any element of $(\mathbb{Z} / n \mathbb{Z})^{*}$. The distribution of $\phi(n)$ and $\lambda(n)$ has been investigated from a variety of perspectives. In particular, many interesting properties of these functions require knowledge of the distribution of prime factors of $\phi(n)$ and $\lambda(n)$ (see e.g., [3]-[7], [12], [19]).

The distribution of all of the divisors of $\phi(n)$ and $\lambda(n)$ has thus far received little attention, perhaps due to the complicated way in which prime factors interact to form divisors. From results about the normal number of prime factors of $\phi(n)$ and $\lambda(n)$ [5], one deduces immediately that $\tau(\phi(n))$ and $\tau(\lambda(n))$ are each about $\exp \left\{\frac{\log 2}{2}(\log \log n)^{2}\right\}$ for almost all $n$. However, the determination of the average size of $\tau(\phi(n))$ and of $\tau(\lambda(n))$ is more complex, and has been studied recently by Luca and Pomerance [13].

In this note we investigate problems about localization of divisors of $\phi(n)$ and $\lambda(n)$. Our results have application to the structure of $(\mathbb{Z} / n \mathbb{Z})^{*}$, since the set of divisors of $\lambda(n)$ is precisely the set of orders of elements of $(\mathbb{Z} / n \mathbb{Z})^{*}$. We say that a positive integer $m$ has $u$-dense divisors (for short, $m$ is $u$-dense) if whenever $1 \leq y<m$, there is a divisor of $m$ in the interval $(y, u y]$. The distribution of $u$-dense numbers for general $u$ has been investigated by Tenenbaum ([17], [18]) and Saias ([14], [15]). According to Théorème 1 of [14], the number of $u$-dense integers $m \leq x$ is $\asymp(x \log u) / \log x$, uniformly for $2 \leq u \leq x$. In particular, the number of 2 -dense integers

2000 Mathematics Subject Classification: Primary 11N64.

Key words and phrases: Euler's function, Carmichael's function, divisors, dense divisors.

First author supported in part by National Science Foundation grant DMS-0555367. 
$m \leq x$ is $\asymp x / \log x$, that is, the 2 -dense integers are about as sparse as the primes.

By contrast, we show that 2-dense values of $\phi(n)$ and $\lambda(n)$ are very common.

TheOREM 1. If $x$ is sufficiently large, then for $\gg x$ integers $n \leq x$, both $\phi(n)$ and $\lambda(n)$ are 2 -dense.

There are relatively simple heuristic reasons for believing Theorem 1. Recall that

$$
\begin{aligned}
\phi\left(p_{1}^{e_{1}} \cdots p_{k}^{e_{k}}\right) & =p_{1}^{e_{1}-1}\left(p_{1}-1\right) \cdots p_{k}^{e_{k}-1}\left(p_{k}-1\right), \\
\lambda\left(p_{1}^{e_{1}} \cdots p_{k}^{e_{k}}\right) & =\operatorname{lcm}\left[\lambda\left(p_{1}^{e_{1}}\right), \ldots, \lambda\left(p_{k}^{e_{k}}\right)\right],
\end{aligned}
$$

where $\lambda\left(p_{i}^{e_{i}}\right)=\phi\left(p_{i}^{e_{i}}\right)$ if $p_{i}$ is odd or if $p_{i}=2$ and $e_{i} \leq 2$, and $\lambda\left(2^{e}\right)=2^{e-2}$ for $e \geq 3$. In particular, $\phi(n)$ and $\lambda(n)$ have the same prime factors. We recall that

- most of these prime factors are factors of shifted primes $p-1$ where $p \mid n$,

- for most primes $p, p-1$ has about $\log \log p$ prime factors [3],

- for most integers $n$, we have $\omega(n)$ about $\log \log n$ and, writing these distinct prime factors as $p_{1}(n)<\cdots<p_{\omega(n)}(n)$, we have for all $k$ except for the smallest ones $\log \log p_{k}(n) \approx k$ (see, e.g., Theorem 10 of [10]),

- $\log \phi(n) \sim \log n$.

With these four facts, we find that for most values of $n$,

$$
\begin{aligned}
\Omega(\phi(n)) & \approx \sum_{p \mid n} \Omega(p-1) \approx \sum_{p \mid n} \log \log p \\
& \approx \sum_{k \leq \log \log n} k \sim(\log \log n)^{2} / 2 \sim(\log \log \phi(n))^{2} / 2 .
\end{aligned}
$$

See [5] for a more precise result about the normal behavior of $\Omega(\phi(n))$. On the other hand, for most values of $m, \Omega(m)$ is about $\log \log m$. So, usually $\phi(n)$ has far more divisors than a typical integer of its size.

We therefore expect the divisors of $\phi(n)$, especially the smaller divisors, to be "very dense" for most $n$, and the same should be true of small divisors of $\lambda(n)$. On the other hand, there are a large proportion of $n$ for which the divisors of $\phi(n)$ and $\lambda(n)$ are not very dense. To state our next result, we define $\theta$ to be the supremum of real numbers $c$ so that there are $\gg x / \log x$ primes $p \leq x$ with $p-1$ having a prime factor $>p^{c}$. Many papers have been written on bounding $\theta$, and the current record is $\theta \geq 0.677$ and is due to Baker and Harman [1]. 
TheOREM 2. Let $0<c<2 \theta-1$. If $x$ is sufficiently large, then for $\gg_{c} x$ of the integers $n \leq x$, neither $\phi(n)$ nor $\lambda(n)$ is $x^{c}$-dense.

It is conjectured that $\theta=1$, and this would imply the conclusion of Theorem 2 for any $c<1$.

If $u<2$, there are no $u$-dense integers $m>1$. However, it is possible that the divisors of a given integer in some long interval do have consecutive ratios which are $\leq u$. We say that an integer $n$ has $u$-dense divisors in a set $I$ (for short, $n$ is $u$-dense in a set $I$ ) if for every $y \in I$, the interval $(y, u y]$ contains a divisor of $n$. The following makes precise what we claimed earlier about the "very dense" nature of the small divisors of $\phi(n)$ and $\lambda(n)$.

TheOREM 3. For every positive integer $h$ and $0<\delta<1$, there is a constant $c=c(h, \delta)>0$ so that if $x$ is sufficiently large, then for more than (1$\delta) x$ of the integers $n \leq x, \phi(n)$ and $\lambda(n)$ are both $(1+1 / h)$-dense in $\left[h, x^{c}\right]$.

Notice that the left endpoint $h$ of the interval cannot be replaced by $h-1$, since if $h-1 \leq a<h /(1+1 / h)$, there are no integers in $(a, a(1+1 / h)]$. In addition, if we assume that $\theta=1$, then we cannot take $c$ independent of $\delta$ in light of Theorem 2.

Using Theorem 3, we prove a more general version of Theorem 1.

TheOREM 4. For every positive integer $h$, there are $\gg_{h} x$ integers $n \leq x$ such that $\phi(n)$ is $(1+1 / h)$-dense in $[h, \phi(n) /(h+1))$ and $\lambda(n)$ is $(1+1 / h)$ dense in $[h, \lambda(n) /(h+1))$.

We also record a limiting case of Theorem 3 .

COROLlary 1. Suppose $g(x)$ is a positive function decreasing monotonically to 0 and let $h$ be a positive integer. Almost all $n \leq x$ have the property that $\phi(n)$ and $\lambda(n)$ are $(1+1 / h)$-dense in $\left[h, x^{g(x)}\right]$.

Analogous to the problems studied in [9], [8], [16], we can study the distribution of integers with $\phi(n)$ having a divisor in a single interval. Let

$$
B(x, y, z)=|\{n \leq x: \exists d \mid \phi(n), y<d \leq z\}| .
$$

An almost immediate corollary of Theorems $1-3$ is the following result in the special case $z=2 y$.

COROllary 2.

(i) Uniformly for $1 \leq y \leq x / 2$, we have $B(x, y, 2 y) \gg x$.

(ii) Fix $1-\theta<c<1 / 2$. Then, uniformly for $x^{c} \leq y \leq x^{1-c}$, we have $x-B(x, y, 2 y) \gg x$.

(iii) Let $g(x) \rightarrow 0$ monotonically. Then, for $1 \leq y \leq x^{g(x)}$, we have $B(x, y, 2 y) \sim x$.

We leave as an open problem the determination of the order of magnitude of $B(x, y, z)$ for all $x, y, z$. 
We note that easy modifications of our proofs give the same results for the sum of divisors function $\sigma(n)$ in place of $\phi(n)$, since $\sigma(p)=p+1$ for primes $p$.

The authors would like to thank Igor Shparlinski for posing the question to study the divisors of $\phi(n)$.

2. Preliminaries. Throughout this paper, the letters $p$ and $q$, with or without subscripts, will always denote primes. Constants implied by the $O$ and $\ll$ symbols are absolute, unless dependence on a parameter is indicated by a subscript. All constants are effectively computable as well. We denote by $P^{+}(m)$ the largest prime factor of $m$, with the convention that $P^{+}(1)=0$.

Our key lemma, presented below, says roughly that the small prime factors of $\phi(n)$ are quite dense.

Lemma 2.1. For some large constant $C$, if $C / \log x \leq g \leq 1 / 10$ and $1 /(g \log x) \leq \varepsilon \leq 1 / 4$, then the number of $n \leq x$ for which $\phi(n)$ does not have a prime divisor in $\left(x^{g}, x^{g(1+\varepsilon)}\right]$ is $\ll g^{\varepsilon / 2} \log (1 / g) x$.

Proof. First, we claim that for large $x$ and $w \geq x^{6 g}$,

$$
\begin{aligned}
& \mid\left\{p \leq w: p-1 \text { has no prime factor in }\left(x^{g}, x^{g(1+\varepsilon)}\right]\right\} \mid \\
& \quad \leq\left(1-\frac{2 \varepsilon}{3}\right) \frac{w}{\log w} .
\end{aligned}
$$

Let $\pi(w ; r, a)$ be the number of primes $p \leq w$ which satisfy $p \equiv a(\bmod r)$. For positive integer $r$, write

where

$$
\pi(w ; r, 1)=\frac{\operatorname{li}(w)}{\phi(r)}+E(w ; r),
$$

$$
\operatorname{li}(w)=\int_{2}^{w} \frac{d t}{\log t} .
$$

Using the Bombieri-Vinogradov theorem ([2, Ch. 28]) and the Mertens estimates, the number of primes $p \leq w$ such that $p-1$ has a prime factor in $\left(x^{g}, x^{g(1+\varepsilon)}\right]$ is

$$
\begin{aligned}
& \geq \sum_{x^{g}<q \leq x^{(1+\varepsilon) g}} \pi(w ; q, 1)-\sum_{x^{g}<q_{1}<q_{2} \leq x^{(1+\varepsilon) g}} \pi\left(w ; q_{1} q_{2}, 1\right) \\
& =\sum_{q}\left(\frac{\operatorname{li}(w)}{q-1}+E(w ; q)\right)-\sum_{q_{1}, q_{2}}\left(\frac{\operatorname{li}(w)}{\left(q_{1}-1\right)\left(q_{2}-1\right)}+E\left(w ; q_{1} q_{2}\right)\right) \\
& =\operatorname{li}(w)\left[\log (1+\varepsilon)-\frac{1}{2} \log ^{2}(1+\varepsilon)+O\left(\frac{1}{\log ^{2} x^{g}}\right)\right]+O\left(\frac{w}{\log ^{3} w}\right) \\
& \geq \frac{3 \varepsilon}{4} \frac{w}{\log w} .
\end{aligned}
$$


For the last step, we used the fact that $w \geq x^{6 g} \geq e^{6 C}$ and $C$ is sufficiently large. This proves (2.1).

Consider $x / \log x<n \leq x$ such that $\phi(n)$ does not have a prime divisor in $\left(x^{g}, x^{g(1+\varepsilon)}\right]$. We can write $n=q_{1}^{\alpha_{1}} \cdots q_{k}^{\alpha_{k}} m$, where $q_{1}>\cdots>q_{k}>x^{6 g}$, $\alpha_{i} \geq 1$ for $1 \leq i \leq k$ and $P^{+}(m) \leq x^{6 g}$. Then $q_{1}, \ldots, q_{k} \in T$, the set of primes $p$ such that $p-1$ does not have a prime factor in $\left(x^{g}, x^{g(1+\varepsilon)}\right]$. By (2.1) and partial summation,

$$
\begin{aligned}
\sum_{\substack{x^{6 g}<q \leq x \\
q \in T}} \sum_{a \geq 1} \frac{1}{q^{a}} & \leq\left(1-\frac{2 \varepsilon}{3}\right)\left(\log \frac{1}{6 g}+\frac{1}{\log x}\right)+\sum_{q>e^{6 C}} \frac{1}{q(q-1)} \\
& \leq\left(1-\frac{\varepsilon}{2}\right) \log \frac{1}{6 g}
\end{aligned}
$$

for sufficiently large $x$. By Theorem 7 of [10], for some positive constant $c_{0}$ and uniformly in $x \geq z, y \geq 2$, the number of integers $n \leq x$ divisible by a number $m>z$ with $P^{+}(m) \leq y$ is $\ll x \exp \left\{-c_{0} \frac{\log z}{\log y}\right\}$. Consequently, the number of $n$ with $m>x^{1 / 3}$ is $\ll x e^{-c_{0} / 18 g} \ll g x$. For other $n$, we may assume that $m \leq x^{1 / 3}$, and thus $k \geq 1$. Again by Theorem 7 of [10], the number of $n$ with $q_{1} \leq \log ^{10} x$ is $\ll x / \log x \ll g x$. For remaining $n$, we have $q_{1}^{\alpha_{1}-1} \cdots q_{k}^{\alpha_{k}-1} \leq \log ^{2} x$, for otherwise, $q_{1}^{\left\lfloor\alpha_{1} / 2\right\rfloor} \cdots q_{k}^{\left\lfloor\alpha_{k} / 2\right\rfloor} \geq$ $q_{1}^{\left(\alpha_{1}-1\right) / 2} \cdots q_{k}^{\left(\alpha_{k}-1\right) / 2}>\log x$ and the number of $n$ divisible by $d^{2}$ for some $d>\log x$ is $O(x / \log x)$. Hence $q_{1} \cdots q_{k} \geq x^{1 / 2}$. In particular, $q_{1} \geq \max \left(x^{1 /(2 k)}\right.$, $\left.\log ^{10} x\right)$ and $\alpha_{1}=1$. Given $q_{2}^{\alpha_{2}}, \ldots, q_{k}^{\alpha_{k}}$, and $m$, the number of $q_{1}$ is, by the Chebyshev estimates for primes,

$$
\ll \frac{x}{q_{2}^{\alpha_{2}} \cdots q_{k}^{\alpha_{k}} m \log \left(x /\left(q_{2}^{\alpha_{2}} \cdots q_{k}^{\alpha_{k}} m\right)\right)} \ll \frac{k x}{\log x} \frac{1}{q_{2}^{\alpha_{2}} \cdots q_{k}^{\alpha_{k}} m} .
$$

Given $q_{2}^{\alpha_{2}}, \ldots, q_{k}^{\alpha_{k}}$

$$
\sum_{P^{+}(m) \leq x^{6 g}} \frac{1}{m} \ll \log \left(x^{6 g}\right)=6 g \log x .
$$

With fixed $k$, we have

$$
\begin{aligned}
\sum_{q_{2}, \ldots, q_{k} \in T} \sum_{\alpha_{2}, \ldots, \alpha_{k} \geq 1} \frac{1}{q_{2}^{\alpha_{2}} \cdots q_{k}^{\alpha_{k}}} & \leq \frac{1}{(k-1) !}\left(\sum_{x^{6 g}<q \leq x} \sum_{\substack{q \in T \\
a \geq 1}} \frac{1}{q^{a}}\right)^{k-1} \\
& \leq \frac{\left(\left(1-\frac{\varepsilon}{2}\right) \log \frac{1}{6 g}\right)^{k-1}}{(k-1) !}
\end{aligned}
$$

The total number of $\operatorname{such} n$ is 


$$
\begin{aligned}
& \ll g x+g x \sum_{1 \leq k \leq 1 /(6 g)} \frac{k}{(k-1) !}\left(\left(1-\frac{\varepsilon}{2}\right) \log \frac{1}{6 g}\right)^{k-1} \\
& \ll g x+g x\left(\log \frac{1}{6 g}\right) \sum_{j=0}^{\infty} \frac{\left(\left(1-\frac{\varepsilon}{2}\right) \log \frac{1}{6 g}\right)^{j}}{j !} \\
& =g x+g x\left(\log \frac{1}{6 g}\right)\left(\frac{1}{6 g}\right)^{1-\varepsilon / 2} \ll g^{\varepsilon / 2}\left(\log \frac{1}{g}\right) x .
\end{aligned}
$$

This completes the proof.

REMARKs. Since $\phi(n)$ and $\lambda(n)$ have the same prime factors, Lemma 2.1 holds with $\phi$ replaced by $\lambda$. With a finer analysis, it is possible to remove the factor $\log (1 / g)$ appearing in the conclusion of Lemma 2.1. Also, if $\varepsilon$ is fixed, then $g^{\varepsilon / 2} \log (1 / g) \ll_{\varepsilon} g^{\varepsilon / 3}$, an inequality we shall use in the application of Lemma 2.1.

We next give a method of constructing integers which are dense in an interval.

Lemma 2.2. Suppose that $h$ is a positive integer, $y \geq h$, and $D$ is $(1+$ $1 / h)$-dense in $[h, y]$. Suppose also that $m=D m_{1} \cdots m_{k}$, where for $1 \leq j \leq k$, $m_{j} \leq(y / h) m_{1} \cdots m_{j-1}$. Then $m$ is $(1+1 / h)$-dense in $\left[h, m_{1} \cdots m_{k} y\right]$.

Proof. By hypothesis, the assertion holds for $k=0$. Suppose the assertion is true for $k=l, m$ satisfies the hypotheses with $k=l+1$, and put $m^{\prime}=D m_{1} \cdots m_{l}$. Then $m^{\prime}$ is $(1+1 / h)$-dense in $\left[h, m_{1} \cdots m_{l} y\right]$. Multiplying the divisors of $m^{\prime}$ by $m_{l+1}$, we find that $m$ is also $(1+1 / h)$-dense in $\left[m_{l+1} h, m_{1} \cdots m_{l+1} y\right]$. Our assumption about $m_{l+1}$ implies that $m$ is $(1+1 / h)$-dense in $\left[h, m_{1} \cdots m_{l+1} y\right]$, as desired.

Lemma 2.3. Given any positive integer $D, n$ is divisible by a prime $q \equiv 1$ $(\bmod D)$ for almost all $n$.

Proof. By a theorem of Landau [11], the number of $n \leq x$ which have no prime factor $q \equiv 1(\bmod D)$ is asymptotic to $c(D) x(\log x)^{-1 / \phi(D)}$ for some constant $c=c(D)$.

Luca and Pomerance [12] have recently proven a stronger statement, namely that for some constant $c_{1}$, for almost all integers $n, \phi(n)$ is divisible by every prime power $\leq c_{1} \frac{\log \log n}{\log \log \log n}$.

\section{Proof of the theorems}

Proof of Theorem 3. Fix $h$ and $\delta$, and let $y$ be sufficiently large, depending on $h$, and such that $y>h^{5}$. Let $D$ be the product of all prime powers $\leq y$. Let $\varepsilon=1 / 4$ and let $Y=(y / h)^{4 / 5}$. Let $C$ be the constant in Lemma 2.1. 
Consider the intervals $I_{j}=\left(Y^{(5 / 4)^{j-1}}, Y^{(5 / 4)^{j}}\right](1 \leq j \leq J)$, where $Y \geq$ $e^{C}$. Fix $c$ so that $0<c \leq 1 / 20$, let $x$ be sufficiently large, and take $J$ so that $Y^{(5 / 4)^{J-2}}<x^{c} \leq Y^{(5 / 4)^{J-1}}$. Then $Y^{(5 / 4)^{J}}<\left(Y^{(5 / 4)^{J-2}}\right)^{2}<x^{2 c} \leq x^{1 / 10}$. By Lemma 2.1, if $y$ is large enough, then the number of integers $n \leq x$ for which $\phi(n)$ does not have prime factors in $I_{j}$ is

$$
\ll\left(\frac{\log Y^{(5 / 4)^{j-1}}}{\log x}\right)^{1 / 12} x
$$

Summing over $j$, we find that $\phi(n)$ has a prime factor in every interval $I_{j}$ for all $n \leq x$ except for a set of size

$$
\ll\left(\frac{\log Y^{(5 / 4)^{J}}}{\log x}\right)^{1 / 12} x<(2 c)^{1 / 12} x .
$$

If $c$ is small enough, for at least $(1-\delta / 2) x$ of the integers $n \leq x, \phi(n)$ has a prime factor in every interval $I_{j}$. By Lemma 2.3 , for at least $(1-\delta) x$ integers $n \leq x, \phi(n)$ is divisible by a prime $q \equiv 1\left(\bmod D^{3}\right)$ and has a prime factor in every interval $I_{j}$. For each such $n$, let $p_{1}, \ldots, p_{J}$ be primes dividing $\phi(n)$ and such that $p_{j} \in I_{j}$ for $1 \leq j \leq J$. By hypothesis, $p_{3}>(y / h)^{5 / 4}>y$, hence $p_{j} \nmid D$ for $j \geq 3$. Since $D^{3}|(q-1)| \lambda(n) \mid \phi(n)$, we see that $\lambda(n)$ and $\phi(n)$ are each divisible by $D p_{1} \cdots p_{J}$. By definition, $D$ is divisible by every positive integer $\leq y$, hence $D$ is $(1+1 / h)$-dense in $[h, y]$. Also, $p_{1} \leq Y^{5 / 4}=y / h$, and for $j \geq 2$,

$$
p_{j} \leq Y^{(5 / 4)^{j}} \leq Y^{5 / 4} \prod_{1 \leq i \leq j-1} Y^{(5 / 4)^{i-1}} \leq(y / h) p_{1} \cdots p_{j-1} .
$$

By Lemma $2.2, \phi(n)$ and $\lambda(n)$ are $(1+1 / h)$-dense in $\left[h, p_{1} \cdots p_{J} y\right]$. Since $p_{J}>Y^{(5 / 4)^{J-1}} \geq x^{c}$, this concludes the proof.

Proof of Theorems 1 and 4. In view of Theorem 3, there is a positive integer $k$ so that when $z$ is large enough, for more than half of the positive integers $d \leq z, \phi(d)$ and $\lambda(d)$ are $(1+1 / h)$-dense in $\left[h, z^{1 / k}\right]$. Put $\varepsilon=$ $1 /\left(5 k^{2}\right)$, let $x$ be sufficiently large and $x^{1 / 2}<d \leq x^{1 / 2+\varepsilon}$, where $\phi(d)$ is $(1+1 / h)$-dense in $\left[h, x^{1 /(2 k)}\right]$. Consider distinct primes $p_{1}, \ldots, p_{k} \in I:=$ $\left[x^{1 /(2 k)-2 \varepsilon}, x^{1 /(2 k)-\varepsilon}\right]$ which do not divide $d$. Note that

$$
x^{1-2 k \varepsilon} \leq d p_{1} \cdots p_{k} \leq x^{1-(k-1) \varepsilon} .
$$

Let $q$ be a prime not dividing $d p_{1} \cdots p_{k}$ and satisfying

$$
\frac{1}{2} \frac{x}{d p_{1} \cdots p_{k}}<q \leq \frac{x}{d p_{1} \cdots p_{k}},
$$

so that by (3.1) and the definition of $\varepsilon$,

$$
x^{1 /(6 k)} \leq q \leq x^{2 /(5 k)} .
$$


We claim that for all such numbers $n=d p_{1} \cdots p_{k} q$ satisfying the additional hypothesis

$$
\lambda(n) \geq x^{1-\varepsilon},
$$

$\phi(n)$ is $(1+1 / h)$-dense in $[h, \phi(n) /(h+1))$ and $\lambda(n)$ is $(1+1 / h)$-dense in $[h, \lambda(n) /(h+1))$. Let $y=x^{1 /(2 k)}$. Note that $\phi(n)=\phi(d)\left(p_{1}-1\right) \cdots\left(p_{k}-1\right)$ $\cdot(q-1), \phi(d)$ is $(1+1 / h)$-dense in $[h, y], p_{i}-1 \leq x^{1 /(2 k)-\varepsilon}<(y / h)(1 \leq i \leq k)$ and $q \leq(y / h)$. By Lemma 2.2 with $D=\phi(d), m_{i}=p_{i}-1(1 \leq i \leq k)$ and $m_{k+1}=q-1, \phi(n)$ is $(1+1 / h)$-dense in $[h, w]$, where $w=y\left(p_{1}-1\right) \cdots\left(p_{k}-1\right)$ $\cdot(q-1)$. By $(3.1)$ and (3.2),

$$
w \geq 2^{-k-1} y p_{1} \cdots p_{k} q \geq 2^{-k-2} \frac{x^{1+1 /(2 k)}}{d} \geq h \sqrt{x} .
$$

But $\phi(n)$ is also $(1+1 / h)$-dense in $[\phi(n) / w, \phi(n) /(h+1))$ since $d \mid m \Leftrightarrow$ $(m / d) \mid m$, and consequently $\phi(n)$ is $(1+1 / h)$-dense in $[h, \phi(n) /(h+1))$.

The argument for $\lambda(n)$ is similar, except that now

$$
\lambda(n)=\lambda(d) \frac{q-1}{f} \prod_{i=1}^{k} \frac{p_{i}-1}{f_{i}},
$$

where $f$ is some divisor of $q-1$ and $f_{i}$ is some divisor of $p_{i}-1(1 \leq i \leq k)$. Here we use (3.4), which implies that $f f_{1} \cdots f_{k} \leq x^{\varepsilon}$. By Lemma 2.2 with $D=\lambda(d), m_{i}=\left(p_{i}-1\right) / f_{i}(1 \leq i \leq k)$ and $m_{k+1}=(q-1) / f$, we see that $\lambda(n)$ is $(1+1 / h)$-dense in $[h, w]$, where

$$
w=y \frac{q-1}{f} \prod_{i=1}^{k} \frac{p_{i}-1}{f_{i}} \geq 2^{-k-2} \frac{x^{1+1 /(2 k)-\varepsilon}}{d} \geq h \sqrt{x} .
$$

As with $\phi(n)$, we conclude that $\lambda(n)$ is $(1+1 / h)$-dense in $[h, \lambda(n) /(h+1))$.

Notice that for the above $n$, when $h=1, \phi(n)$ is 2 -dense in $[1, \phi(n) / 2)$. Since $\phi(n)$ is a divisor of itself, we conclude that $\phi(n)$ is 2 -dense in $[1, \phi(n))$ and hence 2-dense. This conclusion also holds for $\lambda(n)$ by similar arguments.

Finally, we show that the number of such integers $n \leq x$ is $\gg_{h} x$. First, (3.4) holds for almost all $n$ by Theorem 2 of [6]. By the prime number theorem and (3.3), given $d, p_{1}, \ldots, p_{k}$, the number of possible primes $q$ is $\gg_{k} x /\left(d p_{1} \cdots p_{k} \log x\right)$. We also have

$$
\sum_{p_{1}, \ldots, p_{k} \in I} \frac{1}{p_{1} \cdots p_{k}} \gg_{k} 1
$$

and $\sum 1 / d \gg \log x$ by partial summation. Hence, there are $\gg_{k} x$ tuples $\left(d, p_{1}, \ldots, p_{k}, q\right)$ with product $n \in(x / 2, x]$ and with $\phi(n)$ and $\lambda(n)$ being $(1+1 / h)$-dense respectively. Given such an integer $n, n$ has at most $6 k$ prime factors $\geq x^{1 /(6 k)}$, hence the number of tuples $\left(d, p_{1}, \ldots, p_{k}, q\right)$ with product $n$ is bounded by a function of $k$. Thus the proof is complete. 
Proof of Theorem 2. Suppose $0<c<2 \theta-1$, and let $\varepsilon>0$ be so small that $2 \theta-1-6 \varepsilon>c$. Consider $n=p m \leq x$, where $x^{1-2 \varepsilon}<p \leq x^{1-\varepsilon}$, and $P^{+}(p-1)>p^{\theta-\varepsilon}$. By the definition of $\theta$, there are $\gg z / \log z$ such primes $\leq z$, if $z$ is large enough. Then $\phi(n)$ and $\lambda(n)$ are each divisible by a prime $q$ with $q>x^{(1-2 \varepsilon)(\theta-\varepsilon)}>x^{\theta-3 \varepsilon}$, and therefore neither function has divisors in $\left[x^{1-\theta+3 \varepsilon}, x^{\theta-3 \varepsilon}\right]$. The number of such $n$ is, by partial summation,

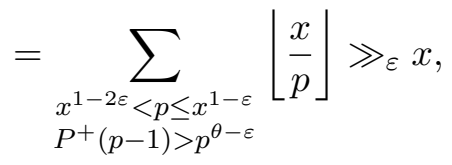

and the proof is complete.

Proof of Corollary 1. Let $\delta>0$. By Theorem 3, if $x$ is sufficiently large, then for at least $(1-\delta) x$ integers $n \leq x$, both $\phi(n)$ and $\lambda(n)$ are $(1+1 / h)$ dense in $\left[h, x^{g(x)}\right]$. Since $\delta$ is arbitrary, the corollary follows.

Proof of Corollary 2. (i) The elementary inequality $\sum_{n \leq x} n / \phi(n) \ll x$ implies that

$$
|\{n \leq x: \phi(n) \leq \varepsilon n\}| \ll \varepsilon x \quad(0<\varepsilon \leq 1) .
$$

Consequently, using Theorem 1 , if $c$ is small enough then there are $\gg x$ integers $n \leq x$ for which $\phi(n)$ is 2 -dense and $\phi(n) \geq c x$. This proves (i) for $y \leq c x$. For a given constant $f \in[c, 1 / 2]$, it is an elementary fact that $f x<\phi(n) \leq 2 f x$ for $\gg_{f} x$ integers $n \leq x$. This completes the proof for the remaining $y$.

(ii) From the proof of Theorem 2, for a positive proportion of integers $n$, $\phi(n)$ has no divisors in $\left[x^{c}, x^{1-c}\right]$.

(iii) This follows immediately from Corollary 1.

\section{References}

[1] R. C. Baker and G. Harman, Shifted primes without large prime factors, Acta Arith. 83 (1998), 331-361.

[2] H. Davenport, Multiplicative Number Theory, 3rd ed., Grad. Texts in Math. 74, Springer, New York, 2000.

[3] P. Erdôs, On the normal number of prime factors of $p-1$ and some related problems concerning Euler's $\phi$-function, Quart. J. Math. Oxford Ser. 6 (1935), 205-213.

[4] P. Erdôs, A. Granville, C. Pomerance, and C. Spiro, On the normal behavior of the iterates of some arithmetic functions, in: Analytic Number Theory (Allerton Park, IL, 1989), Progr. Math. 85, Birkhäuser Boston, Boston, MA, 1990, 165-204.

[5] P. Erdős and C. Pomerance, On the normal number of prime factors of $\phi(n)$, Rocky Mountain J. Math. 15 (1985), 343-352.

[6] P. Erdős, C. Pomerance, and E. Schmutz, Carmichael's lambda function, Acta Arith. 58 (1991), 363-385.

[7] K. Ford, The distribution of totients, Ramanujan J. 2 (1998), 67-151. 
[8] K. Ford, Integers with a divisor in $(y, 2 y]$, in: Anatomy of Integers (Montréal, 2006), CRM Proc. Lecture Notes 46, Amer. Math. Soc., 2008, to appear.

[9] —, The distribution of integers with a divisor in a given interval, Ann. of Math. 168 (2008), 367-433.

[10] R. R. Hall and G. Tenenbaum, Divisors, Cambridge Tracts in Math. 90, Cambridge Univ. Press, Cambridge, 1988.

[11] E. Landau, Lösung des Lehmer'schen Problems, Amer. J. Math. 31 (1909), 86-102.

[12] F. Luca and C. Pomerance, On some problems of Mackowski-Schinzel and Erdôs concerning the arithmetical functions $\phi$ and $\sigma$, Colloq. Math. 92 (2002), 111-130.

[13] - , - On the average number of divisors of the Euler function, Publ. Math. Debrecen 70 (2007), 125-148.

[14] E. Saias, Entiers à diviseurs denses. I, J. Number Theory 62 (1997), 163-191.

[15] —, Entiers à diviseurs denses. II, ibid. 86 (2001), 39-49.

[16] G. Tenenbaum, Sur la probabilité qu'un entier possède un diviseur dans un intervalle donné, Compos. Math. 51 (1984), 243-263.

[17] —, Sur un problème de crible et ses applications, Ann. Sci. École Norm. Sup. (4) 19 (1986), 1-30.

[18] —, Sur un problème de crible et ses applications. II. Corrigendum et étude du graphe divisoriel, ibid. 28 (1995), 115-127.

[19] R. Warlimont, On the iterates of Euler's function, Arch. Math. (Basel) 76 (2001), $345-349$.

Department of Mathematics

University of Illinois

1409 West Green St.

Urbana, IL 61801, U.S.A.

E-mail: ford@math.uiuc.edu cofuns@gmail.com

Received on 2.4.2007

and in revised form on 8.5.2008 